\title{
NUMERICAL STUDY OF TWO-GRID PRECONDITIONERS FOR 1-D ELLIPTIC PROBLEMS WITH HIGHLY OSCILLATING DISCONTINUOUS COEFFICIENTS
}

\author{
O. ILIEV ${ }^{1}$, R. LAZAROV², AND J. WILLEMS 3
}

\begin{abstract}
Various advanced two-level iterative methods are studied numerically and compared with each other in conjunction with finite volume discretizations of symmetric 1-D elliptic problems with highly oscillatory discontinuous coefficients. Some of the methods considered rely on the homogenization approach for deriving the coarse grid operator. This approach is considered here as an alternative to the well-known Galerkin approach for deriving coarse grid operators. Different intergrid transfer operators are studied, primary consideration being given to the use of the so-called problemdependent prolongation. The two-grid methods considered are used as both solvers and preconditioners for the Conjugate Gradient method. The recent approaches, such as the hybrid domain decomposition method introduced by Vassilevski and the globallocal iterative procedure proposed by Durlofsky et al. are also discussed. A two-level method converging in one iteration in the case where the right-hand side is only a function of the coarse variable is introduced and discussed. Such a fast convergence for problems with discontinuous coefficients arbitrarily varying on the fine scale is achieved by a problem-dependent selection of the coarse grid combined with problem-dependent prolongation on a dual grid. The results of the numerical experiments are presented to illustrate the performance of the studied approaches.
\end{abstract}

2000 Mathematics Subject Classification: 65N20, 65M25.

Keywords: two-grid algorithm, oscillating coefficients, preconditioner.

\section{Introduction}

In this paper, we consider the following model problem: find $u(x)$ satisfying

$$
\begin{cases}-\frac{d}{d x}\left(\mu(x) \frac{d u}{d x}\right)=f(x) & \text { in } \Omega \\ u=0 & \text { on } \Gamma\end{cases}
$$

where $\Omega$ is an open interval $(0, \ell)$ and $\Gamma$ denotes its end points. The source $f(x)$ and the coefficient $\mu(x)$ are given functions, which are assumed to be smooth or piecewise constant.

\footnotetext{
${ }^{1}$ Fraunhoferinstitut für Techno- und Wirtschaftsmathematik (ITWM), Fraunhoferplatz 1, 67663 Kaiserslautern, Germany. E-mail: iliev@itwm.fhg.de

${ }^{2}$ Department of Mathematics, Texas A\&M University, College Station, Texas 77843, USA. E-mail: lazarov@math.tamu.edu

${ }^{3}$ Fraunhoferinstitut für Techno- und Wirtschaftsmathematik (ITWM), Fraunhoferplatz 1, 67663 Kaiserslautern, Germany. E-mail: willems@itwm.fhg.de
} 
Additionally, $\mu$ is assumed to be positive and highly varying on a fine scale, so that a coarse scale cannot resolve these variations. It is known (see, e.g., [8]) that (1.1) has a unique weak solution in $H_{0}^{1}(\Omega)$. It follows from the Sobolev inequalities (see, e.g., [8, Chapter 5]) that $u$ is actually continuous. Furthermore, it is known from the regularity theory in [8, Chapter 6 ] that in each subinterval, where $\mu$ and $f$ are constant, $u$ is actually $C^{\infty}$ and satisfies the differential equation (1.1) in a classical sense. Moreover, the flux defined as $W:=-\mu d u / d x$ is continuous throughout the domain $\Omega$.

The continuous problem (1.1) is discretized via finite volumes (see, e.g., [15] or [17, Section 3.3]). The objective of this paper is to analyze numerically the performance of various advanced two-grid methods applied, as solvers and preconditioners, to the discretized problem. The performance of the iterative methods is studied for the difficult case where the coefficient $\mu$ in (1.1) has jump discontinuities and varies over several orders of magnitude on a fine scale, so that discontinuities cannot be resolved by a coarse scale in a multilevel/multigrid algorithm. On the other hand the scale of heterogeneities is still too large to assume the statistically homogeneous case (see, e.g., [11] and [2]). An additional difficulty arises from the fact that unlike most multigrid methods, we consider non-neighboring levels, which resembles the domain decomposition approach. It is known that proper domain decomposition methods, with iteration numbers being independent of the mesh-size and jumps in coefficients, can be written for the case where the domain decomposition resolves discontinuities. Likewise, multigrid methods also work well in the case where the coarse grid resolves the coefficients' discontinuities. On the other hand, various efficient multilevel methods have been developed for problems with fine scale heterogeneities in the case of periodic repetition of a heterogeneous cell. Among these methods are the multigrid and homogenization approach [13], the heterogeneous multiscale method [5], the multiscale finite element methods [1], the multigrid approach [9], and the coarse graining multigrid approach [7] and [6], etc.

The aim of this article is to study the performance of the advanced two-level methods in conjunction with nonperiodic media with fine scale heterogeneities. Those of the above methods, which can be formally applied to nonperiodic media, are adapted for the case of cell-centered finite volume discretizations, and their performance is numerically studied. It should be noted that 1D problems are considered with the aim to concentrate on the multiscale nature of the problem. Most of the considered approaches work also in the multidimensional case, but in this case different efects (e.g., singularity) interefere with each other and make it more difficult to study the pure multiscale effects. On the other hand, we keep in mind that some of the presented here results are specific for the $1 \mathrm{D}$ case. The considered two-grid methods are used both as stand-alone solvers and as preconditioners for the Conjugate Gradient method. Recent approaches, such as the hybrid domain decomposition method from [16] and the global-local iterative procedure from [4] are also discussed. A special two-level method which converges in one iteration is introduced for a special class of problems with varying coefficients.

The goal is to determine algorithmic set-ups such that the number of iterations necessary to achieve a prescribed accuracy is independent of the discretization meshsize and only moderately depends on the magnitude of the jumps of the coefficients. The achievement of the first goal is an inherent property of two-level schemes (see, e.g., [10]). However, this in itself does not guarantee robustness of the methods with respect to the jumps of the coefficients. Our numerical experiments show that this objective is attained when applying problem-dependent inter-grid transfer operators that take into account the fine scale structure of the coefficients of the problem. Depending on its components, the two-grid algorithm, 
can actually define symmetric preconditioners, which we also use in the implementation of the preconditioned conjugate gradient method. In a special case where a problem-dependent coarse grid can be determined, a two-level method converging in one iteration can be designed.

The paper is organized as follows. Some necessary notations are introduced in Section 2 followed by the discretization of problem (1.1) by the finite volume method. A brief outline of a typical two-grid method is also given there. In Section 3, the main components of the two-grid method, namely the smoothing, coarse grid, restriction, and prolongation operators are introduced and discussed. Particular consideration is given to the similarities and differences between the considered approaches. A special two-level method based on the problem-dependent coarse grid is also introduced in this Section. The nodes of the coarse grid in this case are introduced as points at which a fine solution coincides with its value averaged over the considered coarse block. It turns out that the coordinates of these coarse grid nodes depend only on the heterogeneities but not on the boundary conditions. Further on, the next subsection is devoted to the application of the two-grid method as a preconditioner for the Conjugate-Gradient method. In the last subsection, we shortly present the hybrid domain decomposition method proposed by Vassilevski in [16]. Finally, in Section 4, the results of numerical experiments are presented and discussed.

\section{Problem formulation and notations}

2.1. Mesh partitioning and mesh notations. Assume that $\Omega$ is an open interval $(0, \ell)$ and $h \in \mathbb{R}$ is a small parameter such that $0<h \ll \ell$. Also, let us introduce the partition $G_{h}:=\{((j-1) h, j h): j=1, \ldots, m\}$ of $\Omega$ into $m:=\ell / h \in \mathbb{N}$ non-overlapping cells (volumes). Further, let $x_{j}=(j-1 / 2) h, j=1, \ldots, m$ be the centers of the cells in $G_{h}$. Individual blocks of $G_{h}$ will often be called finite volumes and will be denoted by $V_{h}(x)$, where $x=x_{j}$ is the cell center. Additionally, the end points of $\Omega, x=0$ and $x=\ell$, (see Fig. 2.1) are added to the grid and are indexed by $x_{0}=0$ and $x_{m+1}=\ell$, respectively.

Together with the partition $G_{h}$, hereinafter referred to as the fine grid, we also consider the coarse partition, $G_{H}$, where $H$ is chosen to be some multiple of $h$ such that $\ell / H \in \mathbb{N}$. Since $H$ is a multiple of $h$, the faces of the coarse grid cells coincide with the faces of the fine grid, i.e., each coarse cell is an aggregation of a number of fine cells (see Fig. 2.1).

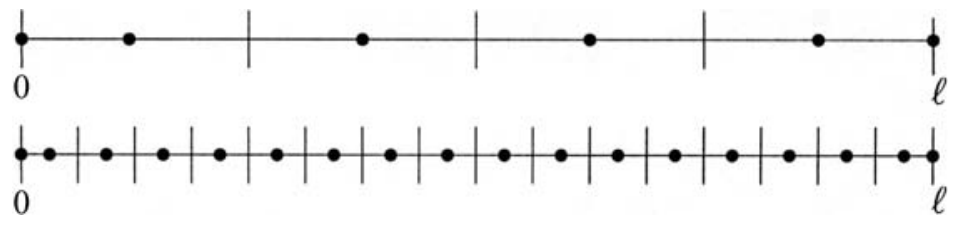

Fig. 2.1. Examples of a coarse and a fine grid on $\Omega$

If there is no danger of confusion, we drop the subindex $h$. However, if necessary, we will include it to indicate that the quantity or the operator in question refers to the fine grid. This will be particularly important in considering the two-grid scheme introduced below. In this case, all quantities and operators with the subindex $H$ will refer to the coarse grid, whereas all variables indexed with $h$ correspond to the fine grid.

Furthermore, we shall need notations for various sets of grid points defined as follows: $\omega:=\left\{x_{j}: 1 \leqslant j \leqslant m\right\}$ is the set of all mesh points inside $\Omega, \stackrel{\circ}{\omega}:=\left\{x_{j}: 2 \leqslant j \leqslant m-1\right\}$ is the set of mesh points that are the centers of cells strictly inside $\Omega, \bar{\omega}_{h}:=\left\{x_{j}: 0 \leqslant j \leqslant m+1\right\}$, all 
mesh points including those on the boundary, and the two near-boundary points $\bar{\gamma}_{h}:=\left\{x_{m}\right\}$, $\underline{\gamma}_{h}:=\left\{x_{1}\right\}$. Additionally, we define $x^{ \pm 1}:=x \pm h$ and $x^{ \pm 1 / 2}:=x \pm h / 2$. Accordingly, we denote $u^{ \pm 1}(x):=u\left(x^{ \pm 1}\right)$ and $u^{ \pm 1 / 2}(x):=u\left(x^{ \pm 1 / 2}\right)$ and analogous expressions for all the other functions that appear.

Further, we introduce the space $\mathscr{U}_{h}$ of the discrete functions defined on $\bar{\omega}_{h}$ and endow it with an inner product

$$
\mathscr{U}_{h}:=\left\{y: \bar{\omega}_{h} \mapsto \mathbb{R}, \text { such that }\left.y\right|_{\bar{\omega}_{h} \backslash \omega_{h}}=0\right\}, \quad(y, z)_{\mathscr{U}_{h}}:=\sum_{x \in \omega_{h}} y(x) z(x) h .
$$

Similarly, we introduce the space $\mathscr{U}_{H}$ of the discrete functions defined on $\bar{\omega}_{H}$ and endow it with an inner product

$$
\mathscr{U}_{H}:=\left\{y: \bar{\omega}_{H} \mapsto \mathbb{R}, \text { such that }\left.y\right|_{\bar{\omega}_{H} \backslash \omega_{H}}=0\right\}, \quad(y, z)_{\mathscr{U}_{H}}:=\sum_{x \in \omega_{H}} y(x) z(x) H
$$

According to these inner products, we define the adjoint of the linear operator $\mathcal{T}_{H \rightarrow h}$ from $\mathscr{U}_{H}$ to $\mathscr{U}_{h}$ by

$$
\left(z_{H}, \mathcal{T}_{H \rightarrow h}^{*} y_{h}\right)_{\mathscr{U}_{H}}:=\left(\mathcal{T}_{H \rightarrow h} z_{H}, y_{h}\right)_{\mathscr{U}_{h}} \quad \forall z_{H} \in \mathscr{U}_{H} \text { and } \forall y_{h} \in \mathscr{U}_{h}
$$

Remark 2.1. If we identify the operators $\mathcal{T}_{H \rightarrow h}$ from $\mathscr{U}_{H}$ to $\mathscr{U}_{h}$ with their corresponding matrices computed with respect to the standard nodal bases in $\mathscr{U}_{H}$ and $\mathscr{U}_{h}$, it is easy to verify that $\mathcal{T}_{H \rightarrow h}^{*}=(h / H) \mathcal{T}_{H \rightarrow h}^{t}$, where $\mathcal{T}_{H \rightarrow h}^{t}$ denotes the transpose of the matrix $\mathcal{T}_{H \rightarrow h}$. This is important for numerical implementations, for it provides an easy way of computing the adjoint of an inter-grid operator.

2.2. Finite volume discretization. We use the standard finite volume discretization of (1.1) as described, e.g., in [15, Chapter 3] or [17, Section 3.3]. To simplify the exposition, we assume that $\mu$ and $f$ are constant on each $V_{h}(x), x \in \omega_{h}$. With

$$
\begin{gathered}
w_{h}^{-1 / 2}(x):=2 \mu(x), \quad \text { for } \quad x \in \underline{\gamma}_{h}, \\
w_{h}^{+1 / 2}(x):=\frac{2 \mu(x) \mu^{+1}(x)}{\mu(x)+\mu^{+1}(x)}, \quad \text { for } \quad x \in \omega \backslash \bar{\gamma}_{h}, \\
w_{h}^{+1 / 2}(x):=2 \mu(x), \quad \text { for } \quad x \in \bar{\gamma}_{h},
\end{gathered}
$$

we may write the discretization of (1.1) as

$$
\mathcal{L}_{h} y=f_{h},
$$

where

$$
\begin{gathered}
\mathcal{L}_{h} y:=\frac{1}{h^{2}} \begin{cases}\left(w_{h}^{-1 / 2}+w_{h}^{+1 / 2}\right) y-w_{h}^{+1 / 2} y^{+1}, & \text { for } x \in \underline{\gamma}_{h}, \\
-w_{h}^{-1 / 2} y^{-1}+\left(w_{h}^{-1 / 2}+w_{h}^{+1 / 2}\right) y-w_{h}^{+1 / 2} y^{+1}, & \text { for } x \in \dot{\omega}_{h}, \\
-w_{h}^{-1 / 2} y^{-1}+\left(w_{h}^{-1 / 2}+w_{h}^{+1 / 2}\right) y, & \text { for } x \in \bar{\gamma}_{h},\end{cases} \\
f_{h}:= \begin{cases}f+w^{-1 / 2} u^{-1 / 2} / h^{2}, & \text { for } x \in \underline{\gamma}_{h}, \\
f, & \text { for } x \in \stackrel{\leftrightarrow}{\omega}_{h}, \\
f+w^{+1 / 2} u^{+1 / 2} / h^{2}, & \text { for } x \in \bar{\gamma}_{h},\end{cases}
\end{gathered}
$$


and $y \in \mathscr{U}_{h}$ denotes the discrete solution. Note that the boundary data $u^{-1 / 2}$ and $u^{+1 / 2}$ in the considered case are zero, but were taken into account in this scheme in order to accommodate the possible nonhomogeneous Dirichlet Boundary Conditions for problem (1.1).

The convergence of scheme (2.2) is established in the following theorem:

Theorem 2.1. Let $u$ be the exact solution of (1.1) and $y$ be the solution of the discrete problem (2.2). If the coefficients $\mu(x)$ and $f(x)$ are piecewise smooth functions on $\Omega$ and the partition into finite volumes is aligned with the jumps of these functions, then $\|u-y\|_{h, \infty}=$ $O\left(h^{2}\right)$ as $h \rightarrow 0$, where $\|y\|_{h, \infty}:=\max _{x \in \bar{\omega}_{h}}|y(x)|$.

Proof. The proof follows the technique presented in [15, Chapter 3]. First, we define the discretization (local truncation) error $\Psi_{h}$ by $\Psi_{h}:=f_{h}-L_{h} u$. Further, for the error $e_{h}$, with $e_{h}\left(x_{h}\right):=u\left(x_{h}\right)-y\left(x_{h}\right) \forall x_{h} \in \bar{\omega}_{h}$, we prove an appropriate a priori estimate in the maximum norm via the local truncation error $\Psi_{h}$. Finally, using the assumption that the solution $y$ is piecewise smooth, we estimate $\Psi_{h}$.

2.3. The two-grid algorithm for the system $\mathcal{L}_{\boldsymbol{h}} \boldsymbol{y}=\boldsymbol{f}_{\boldsymbol{h}}$. Here we present a method for solving the above problem on the basis of two-grid iteration. Obviously, the algebraic system (2.2) can be solved fast and efficiently by a direct method. However, here we would like to test and analyze an iterative method that can also be extended to two- and threedimensional problems.

The two-grid (two-level) method (see, e.g. [3, Chapter 2, p.17] or [10]) is an iterative method for solving the system $\mathcal{L}_{h} y=f_{h}$, which is assumed to be an approximation of the differential equation of (1.1) using finite elements, finite differences, or finite volumes. The method relies on the well-known multigrid approach of error smoothing on the fine grid, computing an approximation to the smoothed error on the coarse grid, and updating the iteration by the computed error. The main ingredients of the two-grid method (and also of the multi-grid method) are:

1) the smoothing operators $\mathcal{S}$ and $\widetilde{\mathcal{S}}$,

2) the coarse-grid operator $\mathcal{L}_{H}$ (in general, some approximation to (1.1) on the coarse grid),

3) the prolongation $\mathcal{T}_{H \rightarrow h}$ and restriction $\mathcal{T}_{h \rightarrow H}$ transfer operators that "communicate" data between the two grids.

On this abstract level the two-grid method can be written as:

Algorithm 2.1 (Two-grid method (TGM)).

1. Assume $y_{h}^{k}$ given.

2. Then set $\operatorname{tgm}\left(y_{h}^{k}, f_{h}\right)=y_{h}^{k+1}$, where $y_{h}^{k+1}$ is defined as follows:

3. $y_{h}^{k+1 / 3}=\mathcal{S}\left(y_{h}^{k}, f_{h}\right)$ (pre-smoothing),

4. $y_{h}^{k+2 / 3}=y_{h}^{k+1 / 3}-\mathcal{T}_{H \rightarrow h} * \mathcal{L}_{H}^{-1} * \mathcal{T}_{h \rightarrow H} *\left(\mathcal{L}_{h} * y_{h}^{k+1 / 3}-f_{h}\right)$ (coarse-grid correction),

5. $y_{h}^{k+1}=\widetilde{\mathcal{S}}\left(y_{h}^{k+2 / 3}, f_{h}\right)$ (post-smoothing).

In this work, we consider two different prolongation and restriction operators and three different coarse-grid operators $\mathcal{L}_{H}$. The restriction operator is often chosen as the adjoint of the prolongation operator. However, it is also interesting to consider the case, where the restriction is not adjoint to the prolongation operator. This case is rarely discussed in the literature (see, e.g., [9, 10,13], etc.). However, as the numerical results in Section 4 show, this approach can be quite reasonable, although some symmetry properties are, in general, lost. In the above algorithm, the solution of the coarse grid system is computed using a direct method. 


\section{Specification of the two-grid algorithm}

We now introduce the main components of Algorithm 2.1 as applied to the discrete problem (2.2).

3.1. Smoothing operators $\mathcal{S}$ and $\widetilde{\mathcal{S}}$. As a smoothing procedure, we simply use the Block - Gauss - Seidel iteration, where the blocks of unknowns correspond to the nodes of the fine grid that lie in one coarse cell (see, e.g., [10, Section 3.3.3]). In general, for symmetry reasons we invert the ordering of the blocks of unknowns in the post-smoothing process and assume that the number of Block - Gauss - Seidel pre-smoothing iterations $n_{\text {pre }}$ and post-smoothing iterations $n_{\text {post }}$ are equal (see, e.g., Section 3.6). However, when this is advantageous, we use $n_{\text {pre }}=0$ and $n_{\text {post }}=1$, compensating for the lost of symmetry by a fast convergence in special cases. We write $\mathcal{S}(y, f)=\mathcal{S}_{\mathrm{BGS} f}\left(y, f, n_{\text {pre }}\right)$ and $\widetilde{\mathcal{S}}(y, f)=$ $\mathcal{S}_{\mathrm{BGSb}}\left(y, f, n_{\text {post }}\right)$.

3.2. Prolongation operator $\mathcal{T}_{\boldsymbol{H} \rightarrow \boldsymbol{h}}$. We introduce two different prolongation operators $\mathcal{T}_{H \rightarrow h}$.

3.2.1. An operator-dependent prolongation operator $\mathcal{T}_{H \rightarrow h}=\mathcal{T}_{H \rightarrow h}^{\mathrm{OPE}}$. Define the operator $\mathcal{T}_{H \rightarrow h}: \mathscr{U}_{H} \mapsto \mathscr{U}_{h}$. Since we are dealing with highly oscillatory discontinuous coefficients $\mu$, the problem-dependent prolongation operator $\mathcal{T}_{H \rightarrow h}=\mathcal{T}_{H \rightarrow h}^{\mathrm{OPE}}$ seems to be a suitable choice (see, e.g., [17, Section 5.4]).

We define the operator-dependent prolongation as follows: given a discrete function $y_{H} \in \mathscr{U}_{H}$, we define $\mathcal{T}_{H \rightarrow h} y_{H}:=\xi_{h}$, where $\xi_{h}$ solves on each coarse grid cell $\left(x_{H}, x_{H}^{+1}\right)$ the discrete problem:

$$
\mathcal{L}_{h} \xi_{h}=0 \quad \text { on } \omega_{h} \cap\left(x_{H}, x_{H}^{+1}\right), \quad \xi_{h}\left(x_{H}\right)=y_{H}\left(x_{H}\right), \quad \xi_{h}\left(x_{H}^{+1}\right)=y_{H}\left(x_{H}^{+1}\right) .
$$

The arising systems are rather small compared to (2.2) and are solved by a direct method. Then we set

$$
\left(\mathcal{T}_{H \rightarrow h}^{\mathrm{OPE}} y_{H}\right)\left(x_{h}\right)=\xi_{h}\left(x_{h}\right), \quad x_{h} \in \omega_{h} \cap\left(x_{H}, x_{H}^{+1}\right) .
$$

Obviously, if the coefficient $\mu(x)$ is constant over the interval $\left(x_{H}, x_{H}^{+1}\right)$, then the prolongation is simply given by the linear interpolation of the values $y_{H}\left(x_{H}\right)$ and $y_{H}\left(x_{H}^{+1}\right)$ on the fine grid.

This type of prolongation operators has a long history and has been used in computational practice (see, e.g., [10] and [17]). The justification for the case of highly oscillatory coefficients can be made rigorous in the periodic case. To illustrate this, we shall present here the analysis carried out in the paper by Neuss et al. in [13].

To this end we need some basic facts from homogenization theory, e.g., [11]. Without loss of generality we assume that $\ell=1$. Furthermore, let $\mu(x)$ be 1-periodic, define $\mu^{\epsilon}(x):=$ $\mu(x / \epsilon), \epsilon \in(0,1]$ being a small parameter. Denote $\langle v\rangle_{(0, \ell)}$ by $\ell^{-1} \int_{0}^{\ell} v d x$ and consider the solution $u^{\epsilon}(x)$ of problem (1.1) with $\mu$ replaced by $\mu^{\epsilon}$.

We now we introduce the auxiliary function $\nu$ as a weak solution of the problem

$$
\left\{\begin{array}{l}
-\frac{d}{d x}\left(\mu \frac{d \nu}{d x}\right)(x)=\frac{d \mu}{d x}(x), \text { for } x \in(0,1), \\
\nu(0)=\nu(1), \\
\mu \frac{d \nu}{d x}(0)=\mu d \frac{d \nu}{d x}(1) .
\end{array}\right.
$$


This is an elliptic b.v.p. with periodic boundary conditions, which has a unique solution $\nu$, such that $\langle\nu\rangle_{(0,1)}=0$. From the homogenization theory (see, e.g., [11,12]) it is wellknown that $u^{\epsilon}$ can be represented as

$$
u^{\epsilon}=u^{0}(x)+\epsilon \nu\left(\frac{x}{\epsilon}\right) \frac{d u^{0}}{d x}(x)+O\left(\epsilon^{2}\right)
$$

where $u^{0}$ is the solution of (1.1) with $\mu$ replaced by $\mu^{0}:=\langle\mu+\mu d \nu(x) / d x\rangle_{(0,1)}$. Here $u^{0}$ represents the large-scale behavior of the solution $u^{\epsilon}$, or the homogenized solution, while the second term, $\epsilon \nu(x / \epsilon) d u^{0}(x) / d x$, is the main oscillatory part.

This structure of the solution $u^{\epsilon}$ of problem (1.1) with a periodic oscillatory coefficient $\mu^{\epsilon}$ suggests the following finite element approximation: (a) solve approximately the corresponding problem for $u^{0}$ to get $u_{H}^{0}$ (e.g., using finite elements on a coarse mesh $\omega_{H}$, with $H=\epsilon$ ); (b) solve approximately the periodic problem (3.3) using finite elements on a fine mesh with a meshsize $h \ll H=\epsilon$ to get the approximation $\nu_{h}^{\epsilon}\left(x_{h}\right)$ to $\nu^{\epsilon}\left(x_{h}\right)$ for $x_{h} \in \omega_{h}$, and finally (c) take

$$
u^{\epsilon}\left(x_{h}\right) \approx u_{H}^{0}\left(x_{h}\right)+\nu_{h}^{\epsilon}\left(x_{h}\right)\left(u_{H}^{0}\left(x_{H}^{+1}\right)-u_{H}^{0}\left(x_{H}\right)\right), \quad x_{h} \in \omega_{h} \cap\left(x_{H}, x_{H}^{+1}\right) .
$$

This result suggests the following procedure for constructing the operator-dependent prolongation used by Fish [9] and Neuss [13] (a similar approach was also used by Eberhard in [6, Section 5.6.1]): assume that $y_{H} \in \mathscr{U}_{H}$ is given on the coarse mesh $\omega_{H}$ with $H=H(\epsilon)=\epsilon$ and choose $h=h(\epsilon) \ll \epsilon$ such that the discontinuities of $\mu^{\epsilon}$ are resolved.

In our setting, $y_{H}$ corresponds to $u_{H}^{0}$ from equation (3.5). The idea of the operatordependent prolongation is to reconstruct the function $\mathcal{T}_{H \rightarrow h}^{\mathrm{OPE}} y_{H} \in \mathscr{U}_{h}$ so that $y_{H}$ represents the large-scale behavior of $\mathcal{T}_{H \rightarrow h}^{\mathrm{OPE}} y_{H}$, i.e., so that (3.5) holds with $u_{H}^{0}$ replaced by $y_{H}$ and $u^{\epsilon}$ replaced by $\mathcal{T}_{H \rightarrow h}^{\mathrm{OPE}} y_{h}$. Since $y_{H}$ is not defined on $\bar{\omega}_{h}$, it is necessary to define first the linear interpolation operator $\mathcal{J}_{h}: \mathscr{U}_{H} \mapsto \mathscr{U}_{h}$ by taking the piecewise linear interpolant between two neighboring coarse grid nodes, say $x_{H}$ and $x_{H}^{+1}$

$$
\left(\mathcal{J}_{h} y_{H}\right)(x):=\frac{x-x_{H}}{H} y_{H}\left(x_{H}^{+1}\right)+\frac{x_{H}^{+1}-x}{H} y_{H}\left(x_{H}\right), \quad \text { for } \quad x \in \omega_{h} \cap\left(x_{H}, x_{H}^{+1}\right) .
$$

Based on the above considerations, Neuss then defines in [13] the prolongation of the discrete function $y_{H}$ in $\mathscr{U}_{H}$ to the function $\mathcal{T}_{H \rightarrow h}^{\mathrm{OPE}} y_{H} \in \mathscr{U}_{h}$ in the following way:

$$
\left(\mathcal{T}_{H \rightarrow h}^{\mathrm{OPE}} y_{H}\right)(x)=\left(\mathcal{J}_{h} y_{H}\right)(x)+\left(y_{H}\left(x_{H}^{+1}\right)-y_{H}\left(x_{H}\right)\right) \nu_{h}^{\epsilon}(x),
$$

where $x \in\left(x_{H}, x_{H}^{+1}\right) \cap \omega_{h}$. Neuss has shown [13] that with this prolongation operator Algorithm 2.1 with no pre-smoothing and one Gauss-Seidel post-smoothing converges with a rate independent of $h$ (if we use the Galerkin approximation for constructing the coarse grid operator, see below).

In the case of piecewise constant periodic coefficients, the operator-dependent prolongation proposed in (3.2) yields the same results as (3.6), as one can readily verify. As opposed to (3.6), however, (3.2) can also be applied with no difficulty to the nonperiodic case. In fact, in the nonperiodic case, although not covered by the analysis above, the operatordependent prolongation in (3.2) yields quite promising numerical results (see, e.g., Section 4). There are two more differences of the proposed procedure as compared to [13]: (1) we use a cell-centered finite volume method to solve approximately fine-grid subproblems and (2) 
the prolongation is defined not on $V_{H}\left(x_{H}\right)$, but on a shifted volume, which is a consequence of considering a cell-centered grid instead of a vertex-centered one.

3.2.2. Trivial prolongation operator $\mathcal{T}_{H \rightarrow h}=\mathcal{T}_{H \rightarrow h}^{\mathrm{CON}}$. The trivial prolongation operator is much simpler than the operator-dependent prolongation. It produces a fine-grid function, whose values at the fine-grid points in a coarse-grid cell equal the value of the coarse-grid function in that cell. More precisely, it is defined as follows:

$$
\left(\mathcal{T}_{H \rightarrow h}^{\mathrm{CON}} y_{H}\right)(x)=y\left(x_{H}\right), \quad \text { for } \quad x \in \omega_{h} \cap V\left(x_{H}\right) .
$$

As would be expected, Algorithm 2.1 using the trivial prolongation operator and its adjoint as a restriction, i.e., $\mathcal{T}_{H \rightarrow h}^{\mathrm{CON}}$, yields rather poor results when applied to problems with oscillating coefficients. Nevertheless, $\mathcal{T}_{H \rightarrow h}^{\mathrm{CON}}$, which can equivalently be defined by taking the volume average over each coarse cell, used together with $\mathcal{T}_{H \rightarrow h}^{\mathrm{OPE}}$ in the two-grid method performs quite promisingly (see, e.g., Section 4).

3.3. Restriction operator $\mathcal{T}_{\boldsymbol{h} \rightarrow \boldsymbol{H}}$. For symmetry reasons, which will be discussed in more detail in Section 3.6, one reasonable approach is to define $\mathcal{T}_{h \rightarrow H}:=\mathcal{T}_{H \rightarrow h}^{*}$, (see (2.1)).

Here, we just note that since $\mathcal{T}_{H \rightarrow h}: \mathscr{U}_{H} \rightarrow \mathscr{U}_{h}$, we have that $\mathcal{T}_{h \rightarrow H}: \mathscr{U}_{h} \rightarrow \mathscr{U}_{H}$, and thus $\mathcal{T}_{h \rightarrow H}$ is a mapping between the desired spaces. We write: $\mathcal{T}_{H \rightarrow h}^{\text {OPE }}=\mathcal{T}_{h \rightarrow H}^{\text {OPE }}$ and $\mathcal{T}_{H \rightarrow h}^{\mathrm{CON}}=\mathcal{T}_{h \rightarrow H}^{\mathrm{CON}}$.

As mentioned above, however, we also consider the case of using the operator-dependent prolongation together with $\mathcal{T}_{h \rightarrow H}^{\mathrm{CON}}$ as a restriction. Unfortunately, this entails loss of some symmetry properties (see, e.g., Section 3.6). Nevertheless, the numerical results of the twogrid method for this case are rather convincing.

3.4. Coarse grid operator $\mathcal{L}_{\boldsymbol{H}}$. For the coarse grid operator $\mathcal{L}_{H}$, we consider three different approaches.

3.4.1. Galerkin approximation, $\mathcal{L}_{H}=\mathcal{L}_{H}^{\mathrm{GA}}$. For this approach we simply set $\mathcal{L}_{H}=$ $\mathcal{T}_{h \rightarrow H} \mathcal{L}_{h} \mathcal{T}_{H \rightarrow h}$, which is suggested, for example, in [10].

3.4.2. Discretization based on numerical upscaling, $\mathcal{L}_{H}=\mathcal{L}_{H}^{\mathrm{HOM}}$. For this approach, the coarse grid operator results from a discretization similar to that discussed in Section 2.2. In the formulae of this Section, we simply replace $\mu$ by

$$
\bar{\mu}\left(x_{H}\right):=\left(\frac{1}{H} \int_{x_{H}^{-1 / 2}}^{x_{H}^{+1 / 2}} \frac{1}{\mu(x)} d x\right)^{-1}=\left(\frac{h}{H} \sum_{x_{h} \in V\left(x_{H}\right)} \frac{1}{\mu\left(x_{h}\right)}\right)^{-1}
$$

and $h$ by $H$. With

$$
\begin{gathered}
\bar{w}_{H}^{-1 / 2}\left(x_{H}\right):=2 \bar{\mu}_{H}\left(x_{H}\right) \quad \text { for } x_{H} \in \underline{\gamma}_{H}, \\
\bar{w}_{H}^{+1 / 2}\left(x_{H}\right):=\frac{2 \bar{\mu}_{H}\left(x_{H}\right) \bar{\mu}_{H}^{+1}\left(x_{H}\right)}{\bar{\mu}_{H}\left(x_{H}\right)+\bar{\mu}_{H}^{+1}\left(x_{H}\right)} \text { for } x_{H} \in \omega_{H} \backslash \bar{\gamma}_{H}, \\
\bar{w}_{H}^{+1 / 2}\left(x_{H}\right):=2 \bar{\mu}_{H}\left(x_{H}\right) \text { for } x \in \bar{\gamma}_{H} .
\end{gathered}
$$

we thus obtain our coarse grid operator as

$$
\mathcal{L}_{H}^{\mathrm{HOM}} y_{H}:=\mathcal{L}_{H} y_{H}
$$

where $\mathcal{L}_{H}$ is defined by (2.3) with $h$ replaced by $H$ and weights determined by (3.8). 
Note that for $\mathcal{L}_{H}^{\mathrm{HOM}}$ the coarse grid permeability in one coarse grid cell is, according to (3.7), given by the harmonic average of the fine grid permeabilities in this coarse cell. In [4], Durlofsky et al. discuss the local-global iteration to determine the upscaled permeability for each coarse grid bock. For this purpose, they present two variants of their iterative procedure (see [4, Section 3.2]). Although Durlofsky's approach seems to be quite promising, for our examples in section 4 it yields almost exactly the same coarse grid permeabilities as (3.7) ( $\ll 1 \%$ difference). Based on this observation, we don't explicitly consider the construction of a coarse grid operator according to [4], since at least for the considered examples this coarse grid operator would be the same as $\mathcal{L}_{H}^{\mathrm{HOM}}$.

3.4.2. "Exact" discretization on the coarse grid, $\mathcal{L}_{H}=\mathcal{L}_{H}^{\mathrm{EXC}}$. The formulae for this case are similar to those of the $\mathcal{L}_{H}^{\mathrm{HOM}}$-case. The only difference is that for $x \in \omega_{H} \backslash \bar{\omega}_{H}$ in (3.8) we replace $\bar{w}_{H}^{+1 / 2}$ by

$$
\widetilde{w}_{H}^{+1 / 2}\left(x_{H}\right)=\left(\frac{1}{H} \int_{x_{H}}^{x_{H}^{+1}} \frac{1}{\mu(x)} d x\right)^{-1}=\left(\frac{h}{H} \sum_{x_{H} \leqslant x_{h} \leqslant x_{H}^{+1}} \frac{1}{\mu\left(x_{h}\right)}\right)^{-1} .
$$

Note, that if the coarse grid resolves the discontinuities of $\mu$, then $\mathcal{L}_{H}^{\mathrm{EXC}}$ is the same as the fine grid operator introduced in Section 2.2 - of course with $h$ replaced by $H$.

Remark 3.1. We can motivate the choices for $\mathcal{L}_{H}^{\mathrm{HOM}}$ and $\mathcal{L}_{H}^{\mathrm{EXC}}$ by the homogenization theory, which is applicable to the case where $\mu$ is periodic (again, we suppose without loss of generality that $\ell=1)$. As noted above, $u^{0}$ in (3.4) is a solution of (1.1) with $\mu$ replaced by $\mu^{0}:=\langle\mu+\mu \partial \nu(x) / \partial x\rangle_{(0,1)}$, where $\nu(x)$ denotes the solution of (3.3). With this one easily obtains $\mu^{0}=\left\langle\mu^{-1}\right\rangle_{(0,1)}^{-1}$. Thus, the homogenized coefficient is given by the harmonic average of the original coefficient. Of course, this is a strong indication that our choices for $\mathcal{L}_{H}^{\text {HOM }}$ and $\mathcal{L}_{H}^{\mathrm{EXC}}$ are quite reasonable.

Note that in [6, section 5.1.1], where the derivation of the numerically upscaled coarse grid operator is based on the coarse graining approach, the same result is obtained for the case of $\mu$ periodic.

3.5. A two-level algorithm with a problem-dependent coarse grid. The considerations in this section are based on the fact that for specific problems surface coordinates, barycentric coordinates, center-of-mass nodes, etc. are introduced in mathematics and physics in order to treat bodies with arbitrary shapes and nonuniform density distributions. Thus, the coarse grid nodes considered here were taken to be the (geometrical) centers of the corresponding coarse grid cells. This is reasonable for homogeneous media or symmetrically heterogeneous cells. Now, we would like to see if it is possible to find such a node within an arbitrary heterogeneous coarse cell that it is more representative of the behavior of the solution in this cell compared to the geometrical center. By analogy, in classical mechanics, to describe the overall motion of a solid body, one has to determine its centerofmass rather than its geometrical center. As a candidate for such a node, we consider a point at which the fine solution of a BVP with arbitrary Dirichlet boundary conditions at the ends of the respective coarse block coincides with its average over this block. As will be shown below, the coordinates of this node do not depend on the choice of the boundary conditions and uniquely depend on the particular fine heterogeneity of this coarse block. Let us formulate these ideas more precisely.

Let $\xi_{H} \in \omega_{H}$ be a coarse grid node and $V_{H}\left(\xi_{H}\right)$ be the corresponding coarse grid volume. Define $\omega_{h, \xi_{H}}:=\omega_{h} \cap V_{H}\left(\xi_{H}\right)$ and $\overline{\omega_{h, \xi_{H}}}:=\omega_{h, \xi_{H}} \cup \partial V_{H}\left(\xi_{H}\right)$. 
Consider now the following coarse cell problem:

$$
\left\{\begin{array}{l}
-\frac{d}{d x}\left(\mu(x) \frac{d u}{d x}\right)=0 \quad \text { in } V_{H}\left(\xi_{H}\right), \\
u\left(\xi_{H}^{-1 / 2}\right)=u_{\xi_{H}^{-1 / 2}}, \quad u\left(\xi_{H}^{+1 / 2}\right)=u_{\xi_{H}^{+1 / 2}}
\end{array}\right.
$$

where $u_{\xi_{H}^{-1 / 2}}, u_{\xi_{H}^{+1 / 2}} \in \mathbb{R}$ are some arbitrarily chosen boundary data with the only restriction that $u_{\xi_{H}^{-1 / 2}} \neq u_{\xi_{H}^{+1 / 2}}$.

Now let

$$
\mathcal{L}_{h, \xi_{H}} y=f_{h, \xi_{H}}
$$

be a discretization of (3.10) formed as described in Section 2.2. Since the solution of (3.10) is piecewise linear and since $\omega_{h}$ resolves the discontinuities of $\mu$, we know by Theorem 2.1 that the discrete solution of (3.11) coincides with the analytical solution of (3.10) in $\omega_{h, \xi_{H}}$.

Let $X\left(\xi_{H}\right) \in V_{H}\left(\xi_{H}\right)$ be such that

$$
u\left(X\left(\xi_{H}\right)\right)=\frac{1}{\# \omega_{h, \xi_{H}}} \sum_{x \in \omega_{h, \xi_{H}}} y(x) .
$$

Since $u$ is piecewise linear, it is easy to see that $X\left(\xi_{H}\right)$ is unique and does not depend on the choice of the boundary data of (3.10), i.e., $u_{\xi_{H}^{-1 / 2}}$ and $u_{\xi_{H}^{+1 / 2}}$. Going through all coarse cells, we obtain for each $\xi_{H} \in \omega_{H}$ exactly one $X\left(\xi_{H}\right)$ satisfying (3.12). Taking into consideration the boundary of our domain $\Omega$, we define $X\left(\xi_{H}\right):=\xi_{H}$, if $\xi_{H} \in\{0, \ell\}$.

Let us assume that $f(x) \equiv f \equiv$ const. $\in \mathbb{R}$. The idea is now to use the just computed $X\left(\xi_{H}\right)$ as new coarse grid nodes and furnish a coarse grid and a coarse grid discretization according to them. To be more precise, we define $\omega_{X, H}:=\left\{X\left(\xi_{H}\right) \mid \xi_{H} \in \omega_{H}\right\}$ and $\overline{\omega_{X, H}}:=$ $\omega_{X, H} \cup\{0, \ell\}$ and the corresponding space $\mathscr{U}_{X, H}:=\left\{y_{X, H}: \overline{\omega_{X, H}} \mapsto \mathbb{R}\right.$, such that $y_{X, H}(0)=$ $\left.u_{0}, y_{X, H}(\ell)=u_{\ell}\right\}$ (here $u_{0}, u_{\ell}$ specify the Dirichlet boundary data of problem (1.1) on $\partial \Omega$ ). Now, we proceed in exactly the same way as in Section 2.2 to derive the stiffness operator $\mathcal{L}_{X, H}: \mathscr{U}_{X, H} \rightarrow \mathscr{U}_{X, H}$ corresponding to the nodal set $\overline{\omega_{X, H}}$. This yields

$$
\mathcal{L}_{X, H} y_{X, H}:=\mathcal{L}_{H} y_{X, H}
$$

where $\mathcal{L}_{H}$ is defined by $(2.3)$ with $h$ replaced by $(X, H)$ and weights given by

$$
\begin{aligned}
w_{X, H}^{-1 / 2}\left(\xi_{H}\right) & :=\left(\int_{\xi_{H}^{-1 / 2}}^{X\left(\xi_{H}\right)}(\mu(x))^{-1}\right)^{-1} \text { for } \xi_{H} \in \underline{\gamma}_{H}, \\
w_{X, H}^{+1 / 2}\left(\xi_{H}\right):= & \left(\int_{X\left(\xi_{H}\right)}^{X\left(\xi_{H}^{+1}\right)}(\mu(x))^{-1}\right)^{-1} \text { for } \xi_{H} \in \omega_{H} \backslash \bar{\gamma}_{H}, \\
w_{X, H}^{+1 / 2}\left(\xi_{H}\right) & :=\left(\int_{X\left(\xi_{H}\right)}^{\xi_{H}^{+\frac{1}{2}}}(\mu(x))^{-1}\right)^{-1} \text { for } \xi_{H} \in \bar{\gamma}_{H} .
\end{aligned}
$$


With this notation the discretized system on the mesh $\omega_{X, H}$ reads

$$
\mathcal{L}_{X, H} y_{X, H}=f_{X, H},
$$

with

$$
f_{X, H}\left(X\left(\xi_{H}\right)\right):=f+ \begin{cases}w_{X, H}^{-1 / 2} y_{X, H}\left(\xi_{H}^{-1 / 2}\right), & \text { for } \xi_{H} \in \underline{\gamma}_{H}, \\ 0, & \text { for } \xi_{H} \in \dot{\omega}_{H}, \\ w_{X, H}^{+1 / 2} y_{X, H}\left(\xi_{H}^{+1 / 2}\right) & \text { for } \xi_{H} \in \bar{\gamma}_{H} .\end{cases}
$$

We now consider Algorithm 2.1 with the components specified as follows:

$$
\begin{gathered}
y_{h}^{0} \equiv 0, \quad \text { i.e., all zero initial guess, } \\
n_{\text {pre }}=0, \quad \text { i.e., no pre-smoothing, } \\
\mathcal{T}_{h \rightarrow H}=\mathcal{T}_{h \rightarrow X, H}^{\mathrm{CON}}, \quad \mathcal{L}_{H}=\mathcal{L}_{X, H}, \quad \mathcal{T}_{H \rightarrow h}=\mathcal{T}_{X, H \rightarrow h}^{\mathrm{OPE}}, \quad \widetilde{\mathcal{S}}=\mathcal{S}_{\mathrm{BGS} b}, \quad \text { with } n_{\text {pre }}=1,
\end{gathered}
$$

where $\mathcal{T}_{h \rightarrow X, H}^{\mathrm{CON}}: \mathscr{U}_{h} \rightarrow \mathscr{U}_{X, H}$ is the restriction operator based on taking the volume averages over each cell corresponding to $\bar{\omega}_{H}$ (and then assigning the average values to the nodes of $\left.\omega_{X, H}\right)$, and $\mathcal{T}_{X, H \rightarrow h}^{\mathrm{OPE}}: \mathscr{U}_{X, H} \rightarrow \mathscr{U}_{h}$ is the problem-dependent prolongation operator defined analogously to $(3.1),(3.2)$ with the grid $\omega_{H}$ replaced by $\omega_{X, H}$.

For all tested numerical examples (see, e.g., Section 4) we can see that after one step of Algorithm 2.1 with components chosen as in (3.16) the achieved accuracy is comparable to that achieved by a direct solver.

3.6. Two-grid iteration as a preconditioner for the conjugate gradient method. We begin this section by stating some wellknown properties of Algorithm 2.1, which are crucial for the application of the two-grid method as a preconditioner of conjugate gradient iteration. In ( $[3$, Section 1.1]) it is discussed that Algorithm 2.1 defines a consistent linear iterative process, i.e., for all $\alpha \in \mathbb{R}$ and $y, z \in \mathscr{U}$ we have

$$
y=\operatorname{tgm}\left(y, \mathcal{L}_{h} y\right)
$$

and

$$
\operatorname{tgm}(y, f)+\alpha * \operatorname{tgm}(z, g)=\operatorname{tgm}(y+\alpha z, f+\alpha g)
$$

provided that $\mathcal{S}$ and $\widetilde{\mathcal{S}}$ have the same properties, i.e., $y=\mathcal{S}(y, L y), y=\widetilde{\mathcal{S}}(y, L y), \mathcal{S}(y, f)+\alpha *$ $\mathcal{S}(z, g)=\mathcal{S}(y+\alpha z, f+\alpha g)$, etc. Note that the Bock — Gauss — Seidel smoothing iterations easily satisfy this requirement.

Properties (3.17) and (3.18) in turn imply the existence of a linear operator $\mathcal{M}_{\mathrm{TGM}}^{-1}$, such that $\operatorname{tgm}(y, f)=y-\mathcal{M}_{\mathrm{TGM}}^{-1}\left(\mathcal{L}_{h} y-f\right)$. The interpretation of this result is that tgm defines an approximate inverse of the stiffness operator $\mathcal{L}_{h}$, namely $\mathcal{M}_{\text {TGM }}^{-1}$, and that the application of $\mathcal{M}_{\text {TGM }}^{-1}$ to some $f$ is equivalent to computing tgm $(0, f)$. In order to use $\mathcal{M}_{\text {TGM }}^{-1}$ as a preconditioner for the Conjugate Gradient Method, we need to make sure that it is symmetric, i.e., self-adjoint with respect to the $(\cdot, \cdot)_{\mathscr{U}}$ inner-product (see, e.g., [14, Section 9]). This follows easily by using the assumptions that the

- the prolongation and restriction operators are adjoint to each other;

- the coarse grid operator is symmetric in the $\mathscr{U}_{H}$-inner-product;

- the number of pre- and post-smoothing steps is the same;

- the ordering of the blocks of unknowns in the post-smoothing iterations is inverted with respect to the ordering of the blocks of unknowns in the pre-smoothing iterations. 
Note that in using $\mathcal{T}_{h \rightarrow H}^{\mathrm{OPE}}$ together with $\mathcal{T}_{h \rightarrow H}^{\mathrm{CON}}$ the first of these assumptions is violated. Due to this fact Algorithm 2.1 does not define a symmetric preconditioner in this case. Nevertheless, it may still be used as a stand-alone solver, and the numerical results in Section 4 show that it is actually quite reasonable to do so.

3.7. Hybrid domain decomposition method. In this section we shortly present the hybrid domain decomposition (DD) method proposed by Vassilevski in [16]. Here it is interesting to compare the numerical results produced by the hybrid DD to those discussed in the previous sections. Let $\mathcal{L}_{h}$ be as in (2.3), and let $M$ be the number of coarse grid cells. In [16], the blocks are formed from fine-grid unknowns lying in one coarse grid cell. Thus, the stiffness matrix is written as

$$
\mathcal{L}_{h}=\left[\begin{array}{ccc}
\mathcal{L}_{h, 11} & \cdots & \mathcal{L}_{h, 1 M} \\
\vdots & \ddots & \vdots \\
\mathcal{L}_{h, M 1} & \cdots & \mathcal{L}_{h, M M}
\end{array}\right]
$$

where $M$ denotes the number of coarse-grid cells. We then choose

$$
\mathcal{B}_{1}=\left[\begin{array}{ccc}
\mathcal{L}_{h, 11}^{-1} & & \\
& \ddots & \\
& & \mathcal{L}_{h, M M}^{-1}
\end{array}\right] .
$$

Now, let $\mathcal{T}$ be the global block diagonal aggregation matrix introduced in [16]. Then $\widetilde{\mathcal{L}_{H}}:=\mathcal{T}^{t} \mathcal{L}_{h} \mathcal{T}$, and we choose $\mathcal{B}_{2}=\widetilde{T} \widetilde{\mathcal{L}_{H}}{ }^{-1} \mathcal{T}^{t}$ according to [16, Eq. (7)]. Then the preconditioner for $\mathcal{L}_{h}$ is chosen to be

$$
\mathcal{M}_{\mathrm{DD}}^{-1}=\mathcal{B}_{1}+\mathcal{B}_{2}\left(I-\mathcal{L}_{h} \mathcal{B}_{1}\right)
$$

which in this setting, according to [16], is equal to $\left(I-\mathcal{B}_{2} \mathcal{L}_{h}\right) \mathcal{B}_{1}\left(I-\mathcal{L}_{h} \mathcal{B}_{2}\right)+\mathcal{B}_{2}$.

\section{Numerical experiments}

This section presents a numerical study of the performance of the approaches discussed above. It will be recalled that earlier multigrid together with homogenization was considered by Fish (in [9]) and Neuss (in [13]). Their considerations were restricted to the periodic case, to considering only the settings when the prolongation and restriction operators are adjoint to each other, and to using the multigrid method as a solver. Our aim is to study the nonperiodic case, as well as to analyze the performance of the two-level methods when they are used as solvers or preconditioners for Krylov subspace methods. As Krylov subspace methods, we consider the GMRES-algorithm and the preconditioned Conjugate Gradient (PCG) method. Since we do not want to go into meticulous detail concerning these methods, we use the built-in Matlab (MATLAB version 6.5.0.180913a Release 13) pcg and gmres functions together with the above preconditioners.

Particular consideration is given here to comparing the results for periodic and nonperiodic problems, studying the influence of the intergrid transfer operators, the choice of the coarse grid operator, etc. Additionally, the two-grid preconditioner for PCG is compared to the two-level DD preconditioner described in the previous section. 
This section is organized as follows. The first subsection includes a description of the test problems studied. The 2nd, 3rd, and 4th subsections present the main results for each of the three tests problems, respectively. The 5th subsection discusses the performance of the two-grid method as a solver and as a preconditioner, mainly for the nonperiodic case. The 6th subsection analyses the influence of the choice of the intergrid transfer operators on the performance of the TGM as a solver and as a preconditioner. The choice of the coarse grid operator is discussed in subsection 7. Subsection 8 is devoted to the comparison of TGM and the two-level DD from the previous section. At the end of this section, a summary of the results obtained is given.

4.1. Test problems. Mainly for the sake of completeness we consider the simplest problem, where the coefficients are in fact constant.

Problem 4.1 (constant coefficients). Consider (1.1) with $\mu(x)=1, f(x)=1$.

Problem 4.2 (periodic coefficients). Let $\mu$ and $f$ of (1.1) be defined by

$$
\mu(x)=\left\{\begin{array}{ll}
1, \quad x \in\left[\frac{2 n}{32}, \frac{2 n+1}{32}\right), & n=0, \ldots, 15, \\
1 \mathrm{e} 4, \quad x \in\left[\frac{2 n-1}{32}, \frac{2 n}{32}\right), & n=1, \ldots, 16,
\end{array} \quad f=1 \text { in } \Omega .\right.
$$

Problem 4.3 (nonperiodic coefficients). Define $f(x)=1$ and

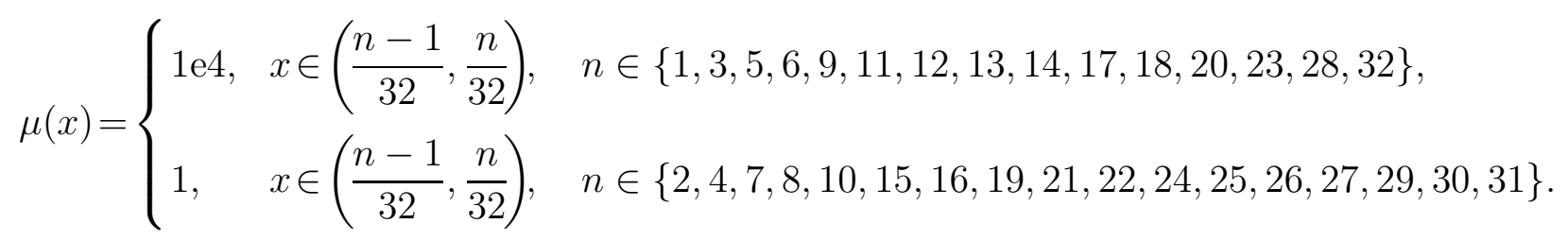

The coefficient $\mu$ is shown in Fig. 4.1 on a log-scale.

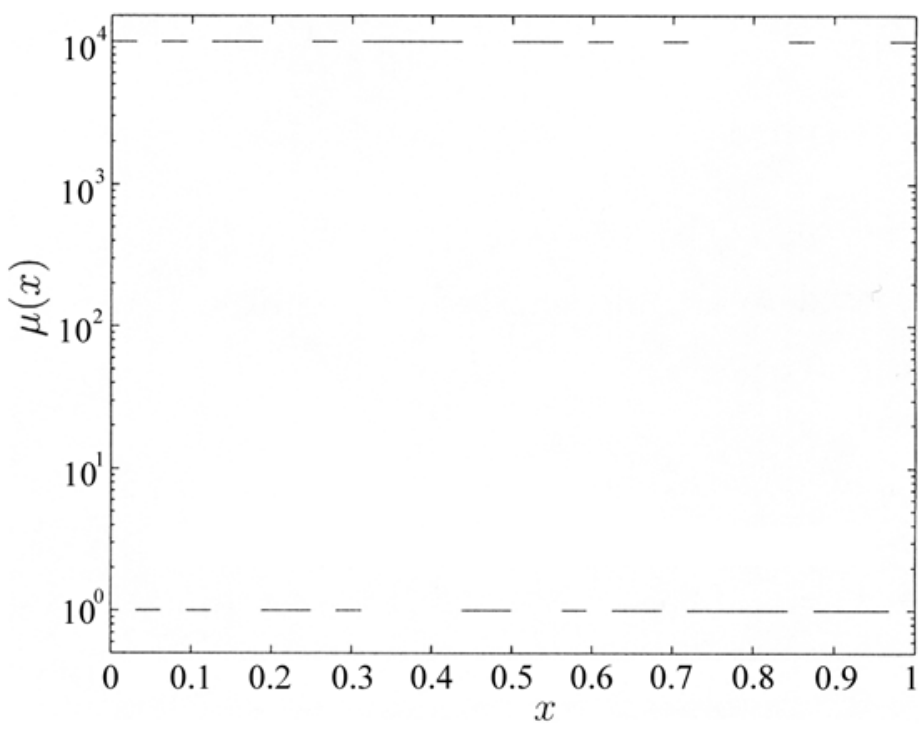

Fig. 4.1. Coefficient of problem 4.3

Note that the jumps of $\mu$ in Problem 4.2 are of the same order of magnitude as the jumps of $\mu$ in Problem 4.3 . 
We choose the number of interior nodes of each grid as some power of 2 , i.e., $\# \omega=$ $2^{k}, k \in \mathbb{N}$ and in the following we will refer to this power as "level". Thus, a grid of level 4 has $2^{4}=16$ interior nodes.

For all iterative processes to be discussed, we choose the initial guess to be zero. (Note that for the DD preconditioner of Section 3.7 this initial guess has to undergo a preprocessing step according to [16, Eq. (9)].) As a stopping criterion, we use the reduction of the $\mathscr{U}_{h}$-norm of the initial residual by a factor of $1 e-6$, where $\|\cdot\|_{\mathscr{U}_{h}}:=\sqrt{(\cdot, \cdot) \mathscr{\mathscr { U }}_{h}}$. It should be noted that the accuracy $1 e-6$ is considered here for two reasons: i) the discretization error may be large for highly varying discontinuous coefficients, and solving the linear system with a high accuracy does not make sense; and ii) to avoid problems with round-off errors, which may appear due to a very large variation of the discontinuous coefficients.

Remark 4.1. Note that for problems 4.1 and 4.2 the coarse grid operators from Sections 4.3 and 3.4 are actually the same, i.e., $\mathcal{L}_{H}^{\mathrm{HOM}}=\mathcal{L}_{H}^{\mathrm{EXC}}$.

4.2. Results for constant coefficients, Problem 4.1. We first we consider the performance of Algorithm 2.1 and the PCG algorithm on Problem 4.1 with $n_{\text {pre }}=n_{\text {post }}=1$. Tables 4.1 and 4.2 display the numerical results.

Table 4.1. Number of TGM iterations needed to reduce the initial residual by a factor of 1e-6 as applied to Problem 4.1

\begin{tabular}{|l|l|c|c|c|c|c|c|c|c|}
\hline \multicolumn{2}{|l|}{ Level of fine grid } & 6 & 7 & 8 & 9 & 10 & 11 & 12 & 13 \\
\hline \multicolumn{2}{|l|}{ Level of coarse grid } & 2 & 3 & 4 & 5 & 6 & 7 & 8 & 9 \\
\hline Operator dependent & $\mathcal{L}_{H}=\mathcal{L}_{H}^{\mathrm{GA}}$ & 36 & 44 & 43 & 41 & 39 & 38 & 37 & 35 \\
prolongation and & $\mathcal{L}_{H}=\mathcal{L}_{H}^{\mathrm{EXC}}$ & 37 & 44 & 43 & 41 & 40 & 38 & 37 & 36 \\
restriction & $\mathcal{L}_{H}=\mathcal{L}_{H}^{\mathrm{HOM}}$ & 37 & 44 & 43 & 41 & 40 & 38 & 37 & 36 \\
\hline Trivial & $\mathcal{L}_{H}=\mathcal{L}_{H}^{\mathrm{GA}}$ & 165 & 233 & 267 & 284 & 295 & 303 & 311 & 318 \\
prolongation and & $\mathcal{L}_{H}=\mathcal{L}_{H}^{\mathrm{EXC}}$ & 16 & 18 & 20 & 21 & 21 & 22 & 22 & 23 \\
restriction & $\mathcal{L}_{H}=\mathcal{L}_{H}^{\mathrm{HOM}}$ & 16 & 18 & 20 & 21 & 21 & 22 & 22 & 23 \\
\hline Operator dependent & $\mathcal{L}_{H}=\mathcal{L}_{H}^{\mathrm{GA}}$ & 19 & 26 & 37 & 40 & 39 & 38 & 37 & 36 \\
prolongation and & $\mathcal{L}_{H}=\mathcal{L}_{H}^{\mathrm{EXC}}$ & 19 & 26 & 37 & 40 & 39 & 38 & 37 & 36 \\
trivial restriction & $\mathcal{L}_{H}=\mathcal{L}_{H}^{\mathrm{HOM}}$ & 19 & 26 & 37 & 40 & 39 & 38 & 37 & 36 \\
\hline
\end{tabular}

Table 4.2. Number of PCG iterations needed to reduce the initial residual by a factor of $1 \mathrm{e}-6$ as applied to Problem 4.1

\begin{tabular}{|l|c|c|c|c|c|c|c|}
\hline Level of fine grid & 6 & 7 & 8 & 9 & 10 & 11 & 12 \\
\hline Level of coarse grid & 2 & 3 & 4 & 5 & 6 & 7 & 8 \\
\hline \# PCG-iterations $\mathcal{L}_{H}^{\mathrm{GA}}, \mathcal{T}_{H \rightarrow h}^{\mathrm{OPE}}, \mathcal{T}_{h \rightarrow H}^{\mathrm{OPE}}$ & 4 & 8 & 9 & 14 & 13 & 13 & 13 \\
\# PCG-iterations $\mathcal{L}_{H}^{\mathrm{EXC}}, \mathcal{T}_{H \rightarrow h}^{\mathrm{OPE}}, \mathcal{T}_{h \rightarrow H}^{\mathrm{OPE}}$ & 4 & 8 & 10 & 14 & 13 & 13 & 13 \\
\# PCG-iterations $\mathcal{L}_{H}^{\mathrm{HOM}}, \mathcal{T}_{H \rightarrow h}^{\mathrm{OPE}}, \mathcal{T}_{h \rightarrow H}^{\mathrm{OPE}}$ & 4 & 8 & 10 & 14 & 13 & 13 & 13 \\
\# PCG-iterations $\mathcal{L}_{H}^{\mathrm{GA}}, \mathcal{T}_{H \rightarrow h}^{\mathrm{CON}}, \mathcal{T}_{h \rightarrow H}^{\mathrm{CON}}$ & 4 & 8 & 16 & 17 & 19 & 21 & 21 \\
\# PCG-iterations $\mathcal{L}_{H}^{\mathrm{EXC}}, \mathcal{T}_{H \rightarrow h}^{\mathrm{CON}}, \mathcal{T}_{h \rightarrow H}^{\mathrm{CON}}$ & 3 & 3 & 3 & 3 & 3 & 3 & 3 \\
\# PCG-iterations $\mathcal{L}_{H}^{\mathrm{HOM}}, \mathcal{T}_{H \rightarrow h}^{\mathrm{CON}}, \mathcal{T}_{h \rightarrow H}^{\mathrm{CON}}$ & 3 & 3 & 3 & 3 & 3 & 3 & 3 \\
\hline
\end{tabular}

Note that in all considered cases the coarse level is obtained by five levels of coarsening the fine level, i.e., each coarse grid cell contains 32 fine grid cells. Furthermore, it should be noted that we do not use Algorithm 2.1 with $\mathcal{T}_{H \rightarrow h}^{\mathrm{OPE}}$ and $\mathcal{T}_{h \rightarrow H}^{\mathrm{CON}}$ as a preconditioner for the PCG algorithm, since in this case the preconditioner $\mathcal{M}_{\text {TGM }}^{-1}$ is not symmetric and thus it does not satisfy the requirements of the PCG-Algorithm. From Tables 4.1 and 4.2 we can see 
that the number of iterations needed for the prescribed residual reduction is asymptotically constant. One more observation from Table 4.1 is rarely discussed in the literature. This is the fact that considering the TGM with trivial prolongation and restriction but with direct discretization on the coarse grid may give better results compared to the use of the Galerkin coarse grid operator.

4.3. Results for piecewise constant periodic coefficients, Problem 4.2. Now, let us focus for a moment on the performance of Algorithm 2.1 as applied to Problem 4.2. We consider two cases where we vary the number of levels between the fine and the coarse level. Table 4.3( $a)$ shows the results for the case where the coarse level is only one level coarser than the fine level and $n_{\text {pre }}=n_{\text {post }}=1$. Table $4.3(b)$ treats the same case except that in this situation the coarse level is five levels coarser than the fine level. In both cases, $n_{\text {pre }}=n_{\text {post }}=1$. From the data collected we deduce that an increase in the difference between the fine and the coarse level leads to an increase in the number of tgm iterations. In fact, it may even happen that the method doesn't even converge (indicated by "Inf") when the fine and the coarse level are five levels apart.

Table 4.3. Number of TGM iterations needed to reduce the initial residual by a factor of 1e-6 as applied to Problem 4.2 with $n_{\text {pre }}=n_{\text {post }}=1$

\begin{tabular}{|l|l|c|c|c|c|c|c|}
\hline \multicolumn{9}{|c|}{$($ a) coarse level = fine level -1} \\
\hline Level of fine grid & 6 & 7 & 8 & 9 & 10 & 11 \\
\hline Level of coarse grid & 5 & 6 & 7 & 8 & 9 & 10 \\
\hline Operator-dependent & $\mathcal{L}_{H}=\mathcal{L}_{H}^{\mathrm{GA}}$ & 6 & 5 & 6 & 6 & 6 & 6 \\
prolongation and restriction & $\mathcal{L}_{H}=\mathcal{L}_{H}^{\mathrm{EXC}}=\mathcal{L}_{H}^{\mathrm{HOM}}$ & 8 & 8 & 8 & 7 & 7 & 7 \\
\hline Trivial prolongation & $\mathcal{L}_{H}=\mathcal{L}_{H}^{\mathrm{GA}}$ & 26 & 26 & 27 & 28 & 28 & 29 \\
and restriction & $\mathcal{L}_{H}=\mathcal{L}_{H}^{\mathrm{EXC}}=\mathcal{L}_{H}^{\mathrm{HOM}}$ & 15 & 10 & 11 & 11 & 11 & 12 \\
\hline Operator dependent prolongation & $\mathcal{L}_{H}=\mathcal{L}_{H}^{\mathrm{GA}}$ & 7 & 7 & 7 & 7 & 7 & 7 \\
and trivial restriction & $\mathcal{L}_{H}=\mathcal{L}_{H}^{\mathrm{EXC}}=\mathcal{L}_{H}^{\mathrm{HOM}}$ & 7 & 7 & 7 & 7 & 7 & 7 \\
\hline
\end{tabular}

\begin{tabular}{|l|l|c|c|c|c|c|c|}
\hline \multicolumn{9}{|c|}{$($ b) coarse level = fine level -5} \\
\hline Level of fine grid & 6 & 7 & 8 & 9 & 10 & 11 \\
\hline Level of coarse grid & 1 & 2 & 3 & 4 & 5 & 6 \\
\hline Operator-dependent & $\mathcal{L}_{H}=\mathcal{L}_{H}^{\mathrm{GA}}$ & 21 & 52 & 79 & 2 & 12 & 20 \\
prolongation and restriction & $\mathcal{L}_{H}=\mathcal{L}_{H}^{\mathrm{EXC}}=\mathcal{L}_{H}^{\mathrm{HOM}}$ & 21 & 52 & 79 & 22 & 12 & 20 \\
\hline Trivial prolongation & $\mathcal{L}_{H}=\mathcal{L}_{H}^{\mathrm{GA}}$ & \multicolumn{7}{|c|}{$>100$} \\
\cline { 2 - 8 } and restriction & $\mathcal{L}_{H}=\mathcal{L}_{H}^{\mathrm{EXC}}=\mathcal{L}_{H}^{\mathrm{HOM}}$ & 11 & 18 & 20 & 22 & Inf $^{*}$ & $>100$ \\
\hline Operator dependent prolongation & $\mathcal{L}_{H}=\mathcal{L}_{H}^{\mathrm{GA}}$ & 21 & 23 & 33 & 2 & 13 & 20 \\
and trivial restriction & $\mathcal{L}_{H}=\mathcal{L}_{H}^{\mathrm{EXC}}=\mathcal{L}_{H}^{\mathrm{HOM}}$ & 21 & 23 & 33 & 22 & 13 & 20 \\
\hline
\end{tabular}

* "Inf" indicates the divergence of the algorithm.

Table 4.4. Number of PCG iterations needed to reduce the initial residual by a factor of $1 \mathrm{e}-6$ as applied to Problem 4.2 with a preconditioner defined by Algorithm 2.1, where $n_{\text {pre }}=n_{\text {post }}=1$ and coarse level $=$ fine level $-\mathbf{5}$

\begin{tabular}{|l|l|c|c|c|c|c|c|}
\hline \multicolumn{2}{|l|}{ Level of fine grid } & 6 & 7 & 8 & 9 & 10 & 11 \\
\hline Level of coarse grid & 1 & 2 & 3 & 4 & 5 & 6 \\
\hline Operator dependent & $\mathcal{L}_{H}=\mathcal{L}_{H}^{\mathrm{GA}}$ & 2 & 4 & 8 & 2 & 5 & 5 \\
prolongation and restriction & $\mathcal{L}_{H}=\mathcal{L}_{H}^{\mathrm{EXC}}=\mathcal{L}_{H}^{\mathrm{HOM}}$ & 2 & 4 & 8 & 4 & 5 & 6 \\
\hline Trivial prolongation & $\mathcal{L}_{H}=\mathcal{L}_{H}^{\mathrm{GA}}$ & 2 & 4 & 8 & 11 & 16 & 10 \\
and restriction & $\mathcal{L}_{H}=\mathcal{L}_{H}^{\mathrm{EXC}}=\mathcal{L}_{H}^{\mathrm{HOM}}$ & 2 & 3 & 3 & 3 & 14 & 8 \\
\hline
\end{tabular}


As for Problem 4.1, we also examine the performance of the PCG-algorithm when used together with $\mathcal{M}_{T G M}^{-1}$, which in this case is a preconditioner induced by the two-grid method, for which the coarse level is five levels coarser than the fine level.

4.4. Results for piecewise constant nonperiodic coefficients, Problem 4.3. Let us now discuss the performance of Algorithm 2.1 as applied to Problem 4.3. We consider several cases, where we vary the number of levels between the fine and the coarse level and the number of smoothing iterations. Tables $4.5(a), 4.5(b)$ correspond to the settings treated in Tables $4.3(a), 4.3(b)$, respectively.

Table 4.5. Number of TGM iterations needed to reduce the initial residual by a factor of $1 \mathrm{e}-6$ as applied to Problem 4.3 with $n_{\text {pre }}=n_{\text {post }}=1$

\begin{tabular}{|l|l|c|c|c|c|c|c|}
\hline \multicolumn{7}{|c|}{$($ a coarse level fine level - 1} \\
\hline \multicolumn{2}{|l|}{ Level of fine grid } & 6 & 7 & 8 & 9 & 10 & 11 \\
\hline \multicolumn{2}{|l|}{ Level of coarse grid } & 5 & 6 & 7 & 8 & 9 & 10 \\
\hline Operator dependent & $\mathcal{L}_{H}=\mathcal{L}_{H}^{\mathrm{GA}}$ & 6 & 6 & 6 & 6 & 6 & 6 \\
prolongation and & $\mathcal{L}_{H}=\mathcal{L}_{H}^{\mathrm{EXC}}$ & 8 & 7 & 7 & 7 & 7 & 7 \\
restriction & $\mathcal{L}_{H}=\mathcal{L}_{H}^{\mathrm{HOM}}$ & 8 & 7 & 7 & 7 & 7 & 7 \\
\hline Trivial & $\mathcal{L}_{H}=\mathcal{L}_{H}^{\mathrm{GA}}$ & 25 & 26 & 27 & 27 & 28 & 29 \\
prolongation and & $\mathcal{L}_{H}=\mathcal{L}_{H}^{\mathrm{EXC}}$ & 13 & 11 & 11 & 11 & 11 & 12 \\
restriction & $\mathcal{L}_{H}=\mathcal{L}_{H}^{\mathrm{HOM}}$ & 13 & 11 & 11 & 11 & 11 & 12 \\
\hline Operator dependent & $\mathcal{L}_{H}=\mathcal{L}_{H}^{\mathrm{GA}}$ & 10 & 7 & 8 & 7 & 7 & 7 \\
prolongation and & $\mathcal{L}_{H}=\mathcal{L}_{H}^{\mathrm{EXC}}$ & 7 & 8 & 7 & 7 & 7 & 7 \\
trivial restriction & $\mathcal{L}_{H}=\mathcal{L}_{H}^{\mathrm{HOM}}$ & 7 & 8 & 7 & 7 & 7 & 7 \\
\hline
\end{tabular}

\begin{tabular}{|l|l|c|c|c|c|c|c|}
\hline \multicolumn{7}{|c|}{$($ b coarse level $=$ fine level -5} \\
\hline \multicolumn{2}{|l|}{ Level of fine grid } & 6 & 7 & 8 & 9 & 10 & 11 \\
\hline Level of coarse grid & 1 & 2 & 3 & 4 & 5 & 6 \\
\hline Operator dependent & $\mathcal{L}_{H}=\mathcal{L}_{H}^{\mathrm{GA}}$ & 16 & 46 & $>100$ & 82 & 47 & 72 \\
prolongation and & $\mathcal{L}_{H}=\mathcal{L}_{H}^{\mathrm{EXC}}$ & 20 & 46 & $>100$ & 73 & 48 & 72 \\
restriction & $\mathcal{L}_{H}=\mathcal{L}_{H}^{\mathrm{HOM}}$ & 20 & 47 & $>100$ & 89 & 48 & 72 \\
\hline Trivial & $\mathcal{L}_{H}=\mathcal{L}_{H}^{\mathrm{GA}}$ & \multicolumn{7}{|c|}{$>100$} \\
\cline { 2 - 9 } prolongation and & $\mathcal{L}_{H}=\mathcal{L}_{H}^{\mathrm{EXC}}$ & 14 & 18 & Inf & Inf & Inf & 74 \\
restriction & $\mathcal{L}_{H}=\mathcal{L}_{H}^{\mathrm{HOM}}$ & 13 & 17 & 20 & Inf & Inf & 54 \\
\hline Operator dependent & $\mathcal{L}_{H}=\mathcal{L}_{H}^{\mathrm{GA}}$ & 16 & 31 & $>100$ & $>100$ & 29 & 36 \\
prolongation and & $\mathcal{L}_{H}=\mathcal{L}_{H}^{\mathrm{EXC}}$ & 20 & 31 & $>100$ & 59 & 29 & 36 \\
trivial restriction & $\mathcal{L}_{H}=\mathcal{L}_{H}^{\mathrm{HOM}}$ & 20 & 32 & 74 & 51 & 29 & 36 \\
\hline
\end{tabular}

* "Inf" indicates the divergence of the algorithm.

Considering the data of Tables $4.5(a), 4.5(b)$ we can again observe that the number of iterations increases with increasing difference between the fine and the coarse level. Again, if the coarse grid is five levels coarser than the fine grid and the trivial prolongation operator is used together with its adjoint as a restriction operator, there are some cases, for which Algorithm 2.1 doesn't converge. The data presented in Table 4.6 show that we can compensate for an increase in the difference between the fine and the coarse level by increasing the number of pre- and post-smoothings. This compensation can be explained by the fact that more smoothings result in a smoother error, whose main characteristics can be captured 
more easily by prolongation operators. In fact, all iterations converge, if we consider the same case as in Table $4.5(b)$ except that now we set $n_{\text {pre }}=n_{\text {post }}=5$ (see, e.g., Table 4.6).

Table 4.6. Number of TGM iterations needed to reduce the initial residual by a factor of $1 \mathrm{e}-6$ when applied to Problem 4.3 with $n_{\text {pre }}=n_{\text {post }}=5$ and coarse level $=$ fine level -5

\begin{tabular}{|l|l|c|c|c|c|c|c|}
\hline Level of fine grid & 6 & 7 & 8 & 9 & 10 & 11 \\
\hline \multicolumn{2}{|l|}{ Level of coarse grid } & 1 & 2 & 3 & 4 & 5 & 6 \\
\hline Operator dependent & $\mathcal{L}_{H}=\mathcal{L}_{H}^{\mathrm{GA}}$ & 11 & 10 & 17 & 12 & 12 & 12 \\
prolongation and & $\mathcal{L}_{H}=\mathcal{L}_{H}^{\mathrm{EXC}}$ & 13 & 10 & 17 & 16 & 12 & 12 \\
restriction & $\mathcal{L}_{H}=\mathcal{L}_{H}^{\mathrm{HOM}}$ & 13 & 10 & 17 & 17 & 12 & 13 \\
\hline Trivial & $\mathcal{L}_{H}=\mathcal{L}_{H}^{\mathrm{GA}}$ & 28 & 86 & \multicolumn{5}{|c|}{$>100$} \\
\cline { 2 - 8 } prolongation and & $\mathcal{L}_{H}=\mathcal{L}_{H}^{\mathrm{EXC}}$ & 10 & 13 & $>100$ & 73 & 17 & 21 \\
restriction & $\mathcal{L}_{H}=\mathcal{L}_{H}^{\mathrm{HOM}}$ & 9 & 13 & 16 & 42 & 17 & 21 \\
\hline Operator dependent & $\mathcal{L}_{H}=\mathcal{L}_{H}^{\mathrm{GA}}$ & 11 & 14 & 19 & 19 & 14 & 15 \\
prolongation and & $\mathcal{L}_{H}=\mathcal{L}_{H}^{\mathrm{EXC}}$ & 13 & 14 & 19 & 16 & 14 & 15 \\
trivial restriction & $\mathcal{L}_{H}=\mathcal{L}_{H}^{\mathrm{HOM}}$ & 12 & 14 & 20 & 17 & 14 & 15 \\
\hline
\end{tabular}

Let us now examine the performance of the PCG algorithm tested with Problem 4.3. As a preconditioner, we use Algorithm 2.1 with $n_{\text {pre }}=n_{\text {post }}=1$ and the coarse level being five levels coarser than the fine level (the case treated in Table 4.5(b)). Table 4.7 shows the results.

Table 4.7. Number of PCG iterations needed to reduce the initial residual by a factor of $1 \mathrm{e}-6$ as applied to Problem 4.3 with a preconditioner defined by Algorithm, 2.1 where $n_{\text {pre }}=n_{\text {post }}=1$ and coarse level $=$ fine level -5

\begin{tabular}{|l|l|c|c|c|c|c|c|}
\hline \multicolumn{2}{|l|}{ Level of fine grid } & 6 & 7 & 8 & 9 & 10 & 11 \\
\hline \multicolumn{2}{|l|}{ Level of coarse grid } & 1 & 2 & 3 & 4 & 5 & 6 \\
\hline Operator dependent & $\mathcal{L}_{H}=\mathcal{L}_{H}^{\mathrm{GA}}$ & 2 & 4 & 8 & 9 & 11 & 17 \\
prolongation and & $\mathcal{L}_{H}=\mathcal{L}_{H}^{\mathrm{EXC}}$ & 2 & 4 & 8 & 10 & 11 & 17 \\
restriction & $\mathcal{L}_{H}=\mathcal{L}_{H}^{\mathrm{HOM}}$ & 2 & 4 & 8 & 13 & 11 & 17 \\
\hline Trivial & $\mathcal{L}_{H}=\mathcal{L}_{H}^{\mathrm{GA}}$ & 2 & 4 & 9 & 17 & 27 & 30 \\
prolongation and & $\mathcal{L}_{H}=\mathcal{L}_{H}^{\mathrm{EXC}}$ & 2 & 4 & 8 & 12 & 17 & 9 \\
restriction & $\mathcal{L}_{H}=\mathcal{L}_{H}^{\mathrm{HOM}}$ & 2 & 4 & 8 & 16 & 17 & 9 \\
\hline
\end{tabular}

As mentioned above, we cannot use Algorithm 2.1 with $\mathcal{T}_{H \rightarrow h}^{\mathrm{OPE}}$ and $\mathcal{T}_{h \rightarrow H}^{\mathrm{CON}}$ as a preconditioner for the PCG algorithm, since in this case the preconditioner is not symmetric. Nonetheless, this preconditioner may in fact be used with GMRES, another well-known Krylov subspace method (see, e.g., [14]). Table 4.8 shows the results when GMRES is applied to the problem discussed in Table 4.7.

T a b le 4.8. Number of GMRES iterations needed to reduce the initial residual by a factor of $1 \mathrm{e}-6$ as applied to Problem 4.3 with a preconditioner defined by Algorithm, 2.1 where $n_{\text {pre }}=n_{\text {post }}=1$ and coarse level $=$ fine level -5

\begin{tabular}{|l|l|c|c|c|c|c|c|}
\hline \multicolumn{2}{|l|}{ Level of fine grid } & 6 & 7 & 8 & 9 & 10 & 11 \\
\hline \multicolumn{2}{|l|}{ Level of coarse grid } & 1 & 2 & 3 & 4 & 5 & 6 \\
\hline \multirow{2}{*}{$\begin{array}{l}\text { Operator dependent } \\
\text { prolongation and }\end{array}$} & $\mathcal{L}_{H}=\mathcal{L}_{H}^{\mathrm{GA}}$ & 2 & 4 & 8 & 12 & 12 & 18 \\
\cline { 2 - 9 } trivial restriction & $\mathcal{L}_{H}=\mathcal{L}_{H}^{\mathrm{EXC}}$ & 2 & 4 & 8 & 12 & 12 & 18 \\
\cline { 2 - 8 } & $\mathcal{L}_{H}=\mathcal{L}_{H}^{\mathrm{HOM}}$ & 2 & 4 & 8 & 14 & 12 & 18 \\
\hline
\end{tabular}


In general, we can say that the results for the GMRESmethod closely resemble those for the PCG-Algorithm. This is of course not very surprising, for we have already seen (see, e.g., Tables 4.5, 4.6) that Algorithm 2.1 used with $\mathcal{T}_{H \rightarrow h}^{\mathrm{OPE}}$ and $\mathcal{T}_{h \rightarrow H}^{\mathrm{CON}}$ is in fact a good stand-alone solver. Its weak point in this case is that its approximate inverse of $\mathcal{L}_{h}$ is not symmetric. Additionally, it should be noted that refining the grid leads to a slight increase in the number of iterations.

The last numerical example is concerned with the performance of the preconditioner mentioned in Section 3.7. In order to compare it to the two-grid preconditioners discussed above, we use it to solve the problem treated in Table 4.7. Table 4.9 shows the results.

T a ble 4.9. Number of PCG iterations needed to reduce the initial residual by a factor of 1e-6 as applied to Problem 4.3 with the hybrid domain decomposition preconditioner defined in Section 3.7

\begin{tabular}{|l|c|c|c|c|c|c|}
\hline Level of fine grid & 6 & 7 & 8 & 9 & 10 & 11 \\
\hline Level of coarse grid & 1 & 2 & 3 & 4 & 5 & 6 \\
\hline PCG with hybrid DD preconditioner & 3 & 5 & 9 & 10 & 9 & 9 \\
\hline
\end{tabular}

4.5. Two-grid method as a solver and as a preconditioner. As can be seen from the Tables above, the number of iterations of the PCG algorithm is much smaller than the number of iterations of Algorithm 2.1 in all cases, which would be expected according to the general theory (see, e.g., [14]). Furthermore, even in the cases where Algorithm 2.1 diverges as a stand-alone solver, it still serves as a good preconditioner for the PCG algorithm.

4.6. Role of the intergrid transfer operators. It appears that in the case of discontinuous coefficients and when the fine and the coarse level are several levels apart, the trivial prolongation operator is just too rough to capture the essential features of the (smoothed) error used to update the iterate. This notion is supported by the fact that an increase in the number of smoothings leads to a converging TGM method for the cases where it diverges with a few smoothings. Apparently, with a sufficiently large number of smoothings the error used to update the iterate is eventually smooth enough to be captured by the trivial prolongation, too.

As opposed to that, the operator-dependent prolongation operator seems to cope rather reliably with an increasing difference between the coarse and the fine level (also for only one pre- and post-smoothing), which is not very surprising, since it actually uses information on the fine-grid-heterogeneities of $\mu$. On the other hand, the TGM method as a solver works equally well for the variants (i) problem-dependent prolongation and its transposed as a restriction, and (ii) problem-dependent prolongation and volume averaging as a restriction. It will be recalled that the latter is less memory consuming compared to the former case. It is expected that in solving nonsymmetric problems (e.g., convection-diffusion equation), variant (ii) could be preferable.

4.7. Role of the coarse-grid operator. The Galerkin approach is widely used to get coarse grid operators in multilevel iterative methods. However, when the coarse operator is obtained using the Galerkin approach starting from the trivial prolongation, it does not reflect well the properties of problems with discontinuous coefficients. When the TGM is used as a solver, it convergences very slowly. In the average, it is still a little bit better than the coarse grid operators built by the other two approaches: homogenization and exact discretization. Surprisingly, the opposite may be observed when the TGM is used as a preconditioner. In this case, the trivial prolongation and restriction work worse with the 
Galerkin coarse grid operator compared to the other two coarse grid operators. Galerkin coarse grid operators also performed worse in using the operator-dependent prolongation (see Table 4.7).

The other two coarse operators, the homogenized one, and so-called exact one, behave very much similarly.

4.8. Role of the problem-dependent coarse-grid. As was mentioned in 3.5, a new coarse grid can be selected in so that the new coarse nodes in each coarse block are points at which a fine solution within a block (with arbitrary Dirichlet data) coincides at this point with the average value of this fine solution. This approach gives extremely good results in the $1 \mathrm{D}$ case, when the right-hand side is zero or when the right-hand side is piecewise constant on the coarse scale. No tables are presented here because for arbitrary choice of discontinuous and highly oscillating on the fine scale coefficients, the TGM from 3.5 converges in 1 sweep. In fact, in the case of a zero right-hand side, using no presmoothing and problem-dependent prolongation, after the first sweep results in an exact solution of the fine problem. In the case where the right-hand side is piecewise constant in the coarse blocks, one has to perform one postsmoothing in order to recover the exact fine solution. This means that the algorithm allows to compute the fine scale solution by applying 2-3 local solvers (within coarse blocks) plus one global solver on the coarse grid. The possibility to extend this approach to the multidimensional case being explored now and will be reported in the future.

4.9. Two-grid preconditioner versus the hybrid DD preconditioner. This subsection concerns comparison of the two-grid preconditioner with the two-level DD preconditioner from section 3.7. The work per iteration on the fine level is comparable for the two approaches when trivial prolongation and restriction are used in the TGM preconditioner. The coarse operator is a little bit more expensive for this particular domain decomposition approach, but the cost for the coarse level operations is low in every way. Comparing Table 4.7 and Table 4.9, one sees that asymptotically both approaches perform in the same way.

4.10. Summary. Considering the data collected, we can draw several conclusions. For constant coefficient problems all methods work well, as would be expected. For the interesting case of nonperiodic jumping coefficients, two cases have to be distinguished. When the TGM is used as a solver, a combination of the homogenized coarse grid operator, the problem-dependent prolongation, and the volume averaging restriction is preferable because of the robust convergence and a lower memory requirement. Note that the other robustly converging variant, with a problem-dependent prolongation and its adjoint as a restriction, requires more memory because the prolongation and the restriction have to be stored in this case. When the TGM was used as a preconditioner for CG, the homogenized coarse grid operator together with problem-dependent prolongation and restriction showed the best results in our numerical study.

Based on the data collected, the homogenized coarse grid operator looks preferable for solving nonperiodic problems with discontinuous coefficients. It should be noted that in the periodic case the homogenized coarse operator and the Galerkin coarse operator, built with a problem-dependent prolongation, are identical. The approach with the problemdependent coarse grid, which allows to converge in one TGM sweep, is the best approach for the considered 1D problems, but unlike the other considered approaches, its generalization to the multidimensional case is not obvious.

Acknowledgements. Part of this research was partially supported by the Kaiserslautern Excellence Cluster Dependable Adaptive Systems and Mathematical Modeling, DASMOD, and by EC under the project INTAS-30-50-4395. 


\section{References}

1. G. Allaire and R Brizzi, A multiscale finite element method for numerical homogenization, Multiscale Model. Simul., 4 (2005), no. 3, pp. 790-812.

2. A. Bourgeat and Piatnitski, A., Approximations of effective coefficients in stochastic homogenization, Ann. Inst. Henri Poincar, Probab. Stat., 40 (2004), no. 2, pp. 153-165.

3. J.H. Bramble, Multigrid Methods, Longman Scientific \& Technical, Essex, 1993.

4. Y. Chen, L. J. Durlofsky, M. Gerritsen, and X. H. Wen, A coupled local-global upscaling approach for simulating flow in highly heterogeneous formations, Advances in Water Resources, 26 (2003), pp. 1041-1060.

5. W.E and B. Engquist, The heterogeneous multi-scale methods, Comm. Math. Sci., 1 (2003), no.1, pp. $87-132$.

6. J. Eberhard, Upscaling und mehrgitterverfahren fur Strömungen in heterogenen porösen Medien, Ruprecht-Karls-Universität Heidelberg, 2003.

7. J. Eberhard, S. Attinger, and G. Wittum, Coarse graining for upscaling of flow in heterogeneous porous media, Multiscale Model. Simul., 2 (2004), no.2, pp. 269-301. 2000 .

8. L. Evans, Partial differential equations, American Mathematical Society, Providence, Rhode Island,

9. J. Fish and V. Belsky, Multigrid methods for periodic heterogeneous media. Part 1: Convergence studies for one-dimensional case, Computer Methods in Applied Mechanics and Engineering, 126 (1995), no. 1-2, pp. 1-16.

10. W. Hackbusch, Multi-grid methods and applications, Springer, Berlin, 2003.

11. V. V. Jikov, S. M. Kozlov, and O. A. Oleinik, Homogenization of differential operators and integral functionals, Springer, Berlin, 1994.

12. D. Lukkassen, G. Nguetseng, and P. Wall, Two-scale convergence, International Journal of Pure and Applied Mathematics, 2 (2002), no. 1, pp. 35-86.

13. N. Neuss, W. Jäger, and G. Wittum, Homogenization and multigrid, Computing, 66 (2001), pp. 1-26.

14. Y.Saad, Iterative methods for sparse linear systems, SIAM, Philadelphia, 2003.

15. A. A. Samarskii, Theory of finite difference schemes, Marcel Dekker, New York, 2001.

16. Y. Vassilevski, A hybrid domain decomposition method based on aggregation, Numerical Linear Algebra with Applications, 11 (2004), no. 4, pp. 327-341.

17. P. Wesseling, An introduction to multigrid methods, Wiley, Chichester, 1992. 